Research Article

\title{
A Biomechanical Comparison of Two Techniques of Latarjet Procedure in Cadaveric Shoulders
}

\author{
Aditya Prinja (D), Antony Raymond, and Mahesh Pimple \\ Whipps Cross University Hospital, London, UK \\ Correspondence should be addressed to Aditya Prinja; a.prinja@cantab.net
}

Received 7 July 2019; Accepted 19 November 2019; Published 24 January 2020

Academic Editor: Benjamin Blondel

Copyright ( $\odot 2020$ Aditya Prinja et al. This is an open access article distributed under the Creative Commons Attribution License, which permits unrestricted use, distribution, and reproduction in any medium, provided the original work is properly cited.

\begin{abstract}
Traumatic anterior instability of the shoulder is commonly treated with the Latarjet procedure, which involves transfer of the coracoid process with a conjoint tendon to the anterior aspect of the glenoid. The two most common techniques of the Latarjet are the classical and congruent arc techniques. The aim of this study was to evaluate the difference in force required to dislocate the shoulder after classical and congruent arc Latarjet procedures were performed. Fourteen cadaveric shoulders were dissected and osteotomised to produce a bony Bankart lesion of $25 \%$ of the articular surface leading to an "inverted pear-shaped" glenoid. An anteroinferior force was applied whilst the arm was in abduction and external rotation using a pulley system. The force needed to dislocate was noted, and then the shoulders underwent coracoid transfer with the classical and congruent arc techniques. The average force required to dislocate the shoulder after osteotomy was $123.57 \mathrm{~N}$. After classical Latarjet, the average force required was $325.71 \mathrm{~N}$, compared with $327.14 \mathrm{~N}$ after the congruent arc technique. This was not statistically significant. In this biomechanical cadaveric study, there is no difference in the force required to dislocate a shoulder after classical and congruent arc techniques of Latarjet, suggesting that both methods are equally effective at preventing anterior dislocation in the position of abduction and external rotation.
\end{abstract}

\section{Introduction}

Recurrent traumatic anterior instability of shoulder is best managed with operative management $[1,2]$. The aim of surgery is to repair the capsule-labral soft tissue structures, and if required, the osseous defects, in order to provide anterior restraint and decrease the capsular volume [3-5]. The Bankart lesion is the most common soft tissue lesion, though variants such as anterior labrum periosteal sleeve avulsion (ALPSA) lesion have been described [6]. Recent arthroscopic techniques have results similar to open procedure with faster rehabilitation and less morbidity [7-10]. However, in the presence of a significant osseous defect, whether humeral (Hill-Sachs lesion) or glenoid (bony Bankart lesion), isolated soft tissue procedures performed either arthroscopic or open have high failure rates [11-14]. The inverse relationship between the size of glenoid defect and the stability of the shoulder has also been established by biomechanical studies [15].
In recent times, addressing the glenoid defect in an attempt to prevent recurrence has gained more attention. The defect can be addressed with a coracoid transfer (the Latarjet procedure), iliac crest bone grafting (the EdenHybinette procedure), or other forms of bone graft such as distal tibial allograft [16-19]. The technique of coracoid transfer, first described by Latarjet in 1954, has undergone many modifications. He described a larger $(2-3 \mathrm{~cm})$ piece of coracoid transferred over to the glenoid rim lengthwise and fixed with 2 screws to create a robust repair [16]. In 1958, Helfet described attaching the raw cut surface of coracoid process to the glenoid neck through the transversely sectioned subscapularis muscle [17]. He named this procedure after his mentor W. Rowley Bristow, who had taught him this surgery nearly two decades prior. Young et al. published his modifications of the procedure, which included the use of 2 screws instead of 1 to provide stable fixation of the coracoid and a subscapularis-splitting approach [20]. The technique was also modified by De Beer et al. who rotated 
the coracoid graft about its long axis to line up the concavity of the coracoid with the articular surface of the glenoid (the so-called "congruent arc" technique) [21].

The aim of this study was to compare the biomechanical efficacy of coracoid transfer using the two common techniques, the "classical" and "congruent arc" Laterjet. Both are well described in literature with good clinical results. We hypothesized that the force needed to dislocate the shoulder would be greater in the congruent arc technique than the classical technique because of increased contact surface area as a result of greater linear dimensions [22, 23].

\section{Materials and Methods}

We dissected 14 cadaveric shoulders. Deltoid and pectoralis major were detached from their clavicular attachment to improve exposure. Subscapularis and anterior capsule were also detached from their insertion on lesser tuberosity. A bony Bankart lesion was created in the anteroinferior rim of the glenoid. The bony lesions were $25 \%$ of longest diameter of the glenoid, to create an "inverted pear-shaped" glenoid (Figure 1) $[15,24]$. The osteotomies were made at a $45^{\circ}$ inclination to the long axis of glenoid, encompassing $\sim 8 \mathrm{~mm}$ width defect of the inferior glenoid circle. The normal shape of glenoid is of a pear when viewed en face, with lower half significantly wider than the upper half. With a large bony Bankart lesion, the upper half become significantly wider than the lower half, resembling the shape of an inverted pear. A hook was inserted in a drill hole in the lateral humerus just inferior to surgical neck. This was passed over a pulley system incorporating a spring balance. The spring balance had a laser marker and a spirit level system attached to it to recreate the direction of force during each application (Figure 2).

The aim was to generate a force directed anteroinferiorly over the humeral head. The arm was kept in 90 degree of abduction and in maximum external rotation. The pulley system was sequentially loaded until the shoulder dislocated anteriorly. The shoulder was said to be dislocated when it would not relocate after releasing the applied force. The force needed to dislocate was noted.

The coracoid tip was then exposed. The insertion of pectoralis minor was detached. The coracoid was osteotomised at the "knee," or the junction of horizontal and vertical parts. The graft was then rigidly fixed flush to the anteroinferior glenoid using the classical technique (Figure 3(a)) with two $3.5 \mathrm{~mm}$ cortical screws, such that the lateral surface of coracoid became the face of the glenoid [16]. The humeral head was then loaded in a manner similar to that used for the native shoulder before coracoid transfer. The force needed to dislocate the shoulder was noted.

The graft was then removed and reoriented according to the congruent arc Latarjet technique, such that the inferior surface of the coracoid becomes the face of the glenoid [21]. Rigid fixation was confirmed with the application of two $3.5 \mathrm{~mm}$ cortical screws. The load was then applied in the similar manner, and the force needed to dislocate shoulder was measured again.

In alternate specimens, the congruent arc Latarjet technique was done first and tested followed by the classical technique. This was done to minimise the effect of any cyclical loading on the biomechanical properties of the construct.

\section{Results}

14 cadaveric specimens were studied. The force required to dislocate uncorrected unstable shoulder was compared with the force required to dislocate the shoulder following "classical" or "congruent arc" Laterjet procedures (Table 1).

A paired $t$-test was used to calculate the difference in mean force needed to dislocate shoulder, before and after the coracoid transfer. The force was calculated with the formula, $F($ in Newton $)=$ load $\times$ gravity .

The mean force required to dislocate the shoulder after the classical Latarjet was $325.71 \mathrm{~N}$ compared with $123.57 \mathrm{~N}$ in the uncorrected shoulder. The standard errors of mean and standard deviation were $6.51 \mathrm{~N}$ and $24.37 \mathrm{~N}$, respectively, in uncorrected shoulders. The standard errors of mean and standard deviation were $8.30 \mathrm{~N}$ and $31.06 \mathrm{~N}$, respectively, for the shoulders undergoing the classical technique. $95 \%$ confidence interval was from -209.00 to -195.28 . The two-tailed $P$ value was less than 0.0001 , thus the difference was statistically significant.

The mean force required to dislocate the shoulder after the congruent arc Laterjet was $327.14 \mathrm{~N}$ compared with 123.57 $\mathrm{N}$ in the uncorrected shoulder. The standard errors of mean and standard deviation were $6.51 \mathrm{~N}$ and $24.37 \mathrm{~N}$, respectively, in uncorrected shoulders, whereas, the standard errors of mean and standard deviation were $7.94 \mathrm{~N}$ and $29.72 \mathrm{~N}$, respectively, in the shoulder treated with the congruent arc technique. 95\% confidence interval was from -214.57 to -192.57 . The two-tailed $P$ value was less than 0.0001 , thus the difference was statistically significant.

An unpaired $t$-test was performed to compare the force required to dislocate the shoulder treated with the two different techniques. Mean force required to dislocate the shoulder after the classical technique was $325.7 \mathrm{~N}$ compared with $327 \mathrm{~N}$ after the congruent arc technique. The two-tailed $P$ value equals 0.9020 and the $95 \%$ confidence interval from -25.05 to 22.19 , thus the difference was not statistically significant.

\section{Discussion}

The optimal strategy for surgical stabilization of the unstable glenohumeral joint remains controversial. However, there seems to be an increased awareness that an isolated soft tissue procedure alone is not always appropriate, and addressing osseous defects of a significant size is important to ensure biomechanical stability and good clinical outcomes $[13,25,26]$. The rationale for the Latarjet procedure is that firstly, it provides a "bone block" to fill the void of an anteroinferior glenoid defect and increases the contact surface area of the glenohumeral articulation. Secondly, and crucially, a sling is created by the dynamic support of the repositioned conjoint tendon which supports the humeral head and provides increased stability in abduction and external rotation (the so-called "dynamic sling effect") [27-30]. 


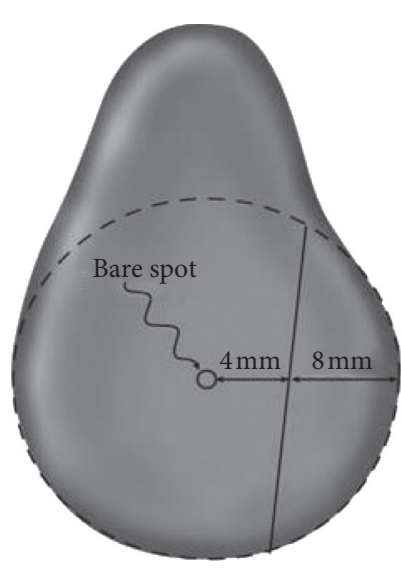

(a)

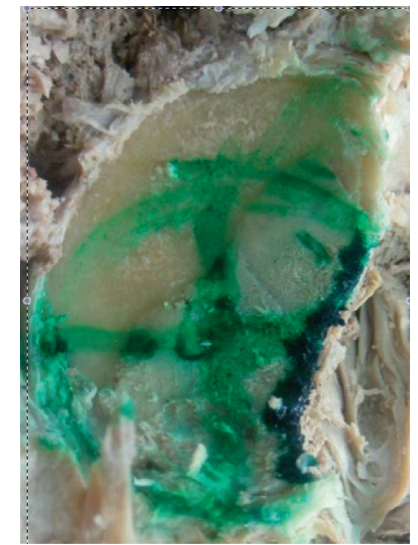

(b)

FIgURE 1: Osteotomy of cadaveric glenoid to recreate "inverted pear-shaped" anterior bony defect. (a) Posterior. (b) Anterior.

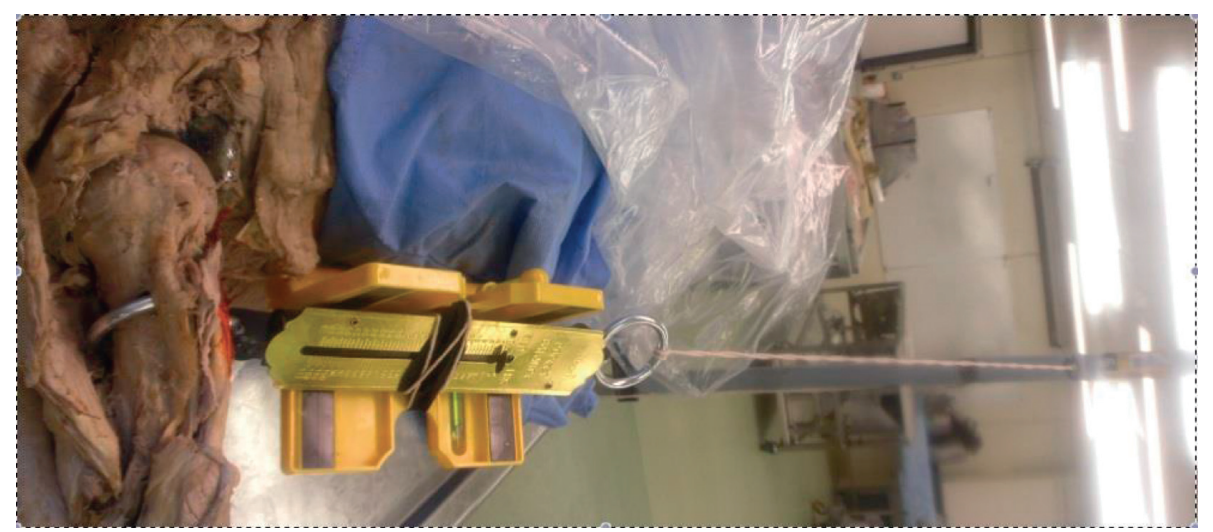

Figure 2: Pulley system attached to the cadaveric upper arm to recreate dislocation force.

The repair of anterior capsulolabral structures has been the standard treatment for traumatic anterior shoulder, since the essential lesion was first described by Perthes, and later by Bankart [31-33]. In Bankart's series of 27 patients, none had bony involvement, leading him to postulate that this was in fact a rare combination [32]. Later, however, Rowe showed in a series of 158 patients, almost three quarters had glenoid rim involvement [33]. The open Bankart procedure, restoring near normal anatomy with low recurrence rates, had been recognized as the gold standard treatment for many years, although the functional outcomes were sometimes reported as suboptimal [34-36]. The arthroscopic Bankart repair was subsequently introduced with the aim of decreasing the morbidity and improving functional outcomes. Despite mixed results initially, advances in arthroscopic techniques have led to widespread uptake of the procedure with good results [8,37-39]. However, several studies have shown increased rates of failures for the arthroscopic procedures, where the significant osseous defects were not addressed $[13,24,25,40,41]$. In their study of 194 arthroscopic Bankart repairs, Burkhart et al. [13] reported a $4 \%$ recurrence rate in patients without significant bony defects compared with a $67 \%$ recurrence rate in those with a significant bony defect (7\% vs.
$89 \%$ in contact athletes, respectively). The study cited a failure to adequately address the glenohumeral osseous defect as the main cause of recurrence.

The glenohumeral defect can be addressed with various methods of coracoid transfer as previously mentioned. In this study, we compared the two most commonly utilised techniques, the "classical" and "congruent arc" Latarjet. In the congruent arc technique, the coracoid graft is rotated about its long axis, and the concavity is lined up with the joint surface [22]. This relatively increases the anteroposterior diameter and hence increases the surface area for anterior translation in comparison with the classic Latarjet technique, where the inferior surface of the coracoid sits on to the anterior inferior rim of glenoid. Furthermore, it has been shown that the coracoid transfer performed using the congruent arc technique restores the glenohumeral loading mechanics to intact condition, while the classical technique restores it within $5 \%$ of the intact state [23]. We hypothesized that this relative increase in surface area would make the bony block with the congruent arc technique, a more stable construct in comparison with the classical latarjet technique. This was expected to reflect as an increase in force requirement for dislocation. However, the difference in force 


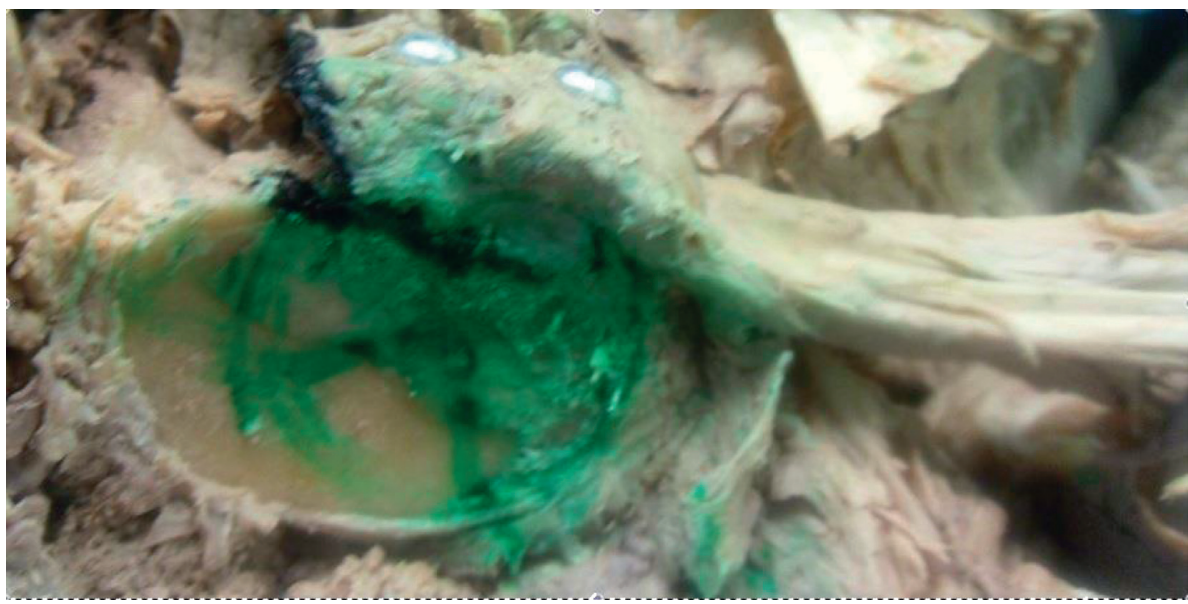

(a)

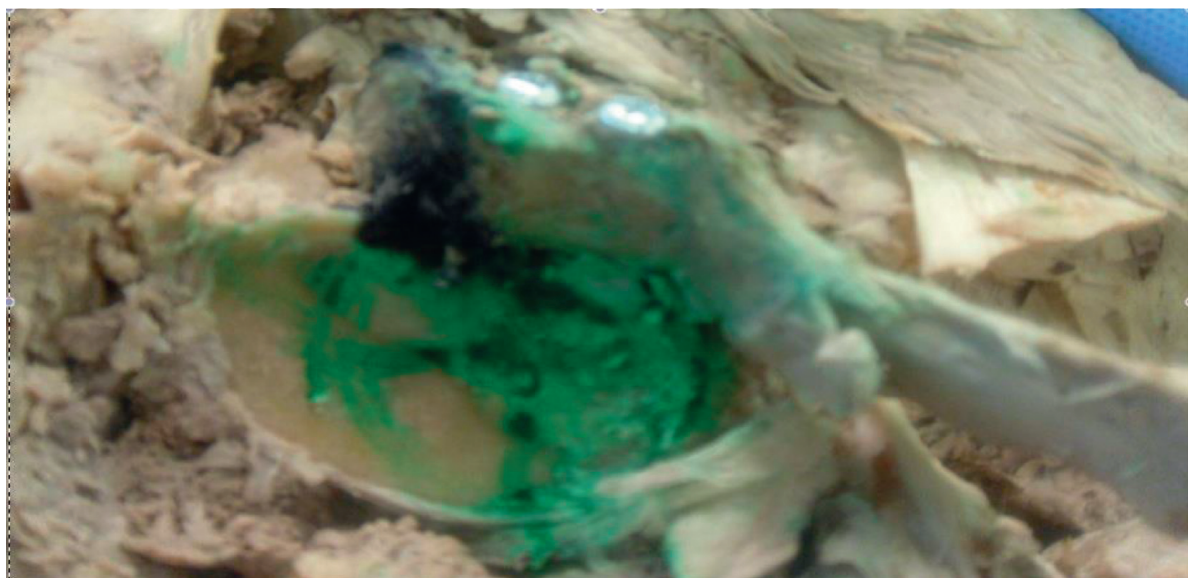

(b)

Figure 3: (a) The classical Latarjet. (b) The congruent arc Latarjet.

TABLE 1: Load required to dislocate the shoulder pre- and postcoracoid transfer (with the two techniques).

\begin{tabular}{|c|c|c|c|}
\hline \multirow[b]{2}{*}{ Cadaver } & \multicolumn{3}{|c|}{ Load required to dislocate $(\mathrm{kg})$} \\
\hline & $\begin{array}{c}\text { After } \\
\text { osteotomy }\end{array}$ & $\begin{array}{l}\text { Classical } \\
\text { Latarjet }\end{array}$ & $\begin{array}{c}\text { Congruent arc } \\
\text { Latarjet }\end{array}$ \\
\hline 1 & 12 & 32 & 33 \\
\hline 2 & 17 & 39 & 36 \\
\hline 3 & 9 & 29 & 31 \\
\hline 4 & 15 & 36 & 37 \\
\hline 5 & 13 & 32 & 31 \\
\hline 6 & 11 & 28 & 30 \\
\hline 7 & 16 & 37 & 38 \\
\hline 8 & 13 & 33 & 31 \\
\hline 9 & 10 & 30 & 29 \\
\hline 10 & 11 & 32 & 32 \\
\hline 11 & 10 & 30 & 30 \\
\hline 12 & 12 & 33 & 30 \\
\hline 13 & 10 & 31 & 34 \\
\hline 14 & 14 & 34 & 36 \\
\hline
\end{tabular}

needed to dislocate in these two techniques was not statistically significant to establish the superiority of one procedure over the other.
In another cadaveric study by Montgomery et al. significant different loads to failure for the two types of coracoid transfer were demonstrated [42]. They found that the congruent arc technique resulted in a lower mean failure load as compared with the classic technique; however, they were applying a tensile load to the conjoint tendon in a bid to replicate the forces experienced by the graft in the early postoperative period. They remarked that the classic technique created a larger surface area for healing to the native glenoid, whilst the congruent arc produced a greater surface area of the glenoid articular surface. They said, as a result, individual patients' anatomy should be preoperatively considered prior to selecting a technique. Giles et al. in their cadaveric comparison of the two techniques applied medially directed forces across the transferred coracoid to try and replicate the forces across the glenohumeral joint and found that the classic technique failed at a higher load than the congruent arc [43].

Mook et al. demonstrated that assessment of coracoid size preoperatively could predict outcome after Latarjet [44]. They suggested that if predicted glenoid track remained offtrack with a classically performed Latarjet, a congruent arc might prove beneficial with its larger surface area. Others 
have said, however, that larger grafts than necessary will see higher rates of graft osteolysis, as less forces from the humeral head are applied leading to resorption in accordance with Wolff's law [45].

In this study, our results show that there is no statistically significant difference in the force required to produce an anteroinferior dislocation of the shoulder after either classical or congruent arc Latarjet. This suggests that both techniques will provide adequate bony coverage to an anterior glenoid defect and will be effective in preventing recurrent dislocation.

Limitations must be considered when interpreting our results. Firstly, this study was performed on cadavers with a mean age of 84.6 years. This is a procedure most commonly performed on patients who are much younger, and thus the effect of reduced bone mineral density of the grafted coracoid could have affected results. Furthermore, this study did not consider the effect of the conjoint tendon and the dynamic sling effect, and it may be due to the fact that tendon has differing effects according to the position, nor did it consider other soft tissue factors such as capsule-labral repair or the subscapularis split. In this study, we did not aim to address the issue of union of the coracoid, which may also be different, as the techniques differ in the area that is in contact with the glenoid. However, in this study, failure of the construct was solely due to failure of the coracoid transfer itself, and the study has clearly demonstrated that there is no difference in the performance of the transfer using the two techniques.

\section{Conclusion}

To conclude, both the congruent arc and classical technique of coracoid transfer are equally effective in preventing anterior shoulder dislocation in the position of abduction and external rotation in cadaveric specimens.

\section{Data Availability}

The data used to support the findings of this study are available from the corresponding author upon request.

\section{Conflicts of Interest}

The authors declare that they have no conflicts of interest.

\section{References}

[1] T. R. Lenters, A. K. Franta, F. M. Wolf, S. S. Leopold, and F. A. Matsen III, "Arthroscopic compared with open repairs for recurrent anterior shoulder instability," The Journal of Bone \& Joint Surgery, vol. 89, no. 2, pp. 244-254, 2007.

[2] S. Pelet, B. M. Jolles, and A. Farron, "Bankart repair for recurrent anterior glenohumeral instability: results at twentynine years' follow-up," Journal of Shoulder and Elbow Surgery, vol. 15, no. 2, pp. 203-207, 2006.

[3] D. W. Altcheck, R. F. Warren, M. J. Skyhar, and G. Ortiz, “Tplasty modification of the Bankart procedure for multidirectional instability of the anterior and inferior types," The Journal of Bone \& Joint Surgery, vol. 73-A, pp. 105-112, 1991.
[4] C. S. Neer and C. R. Foster, "Inferior capsular shift for involuntary inferior and multidirectional instability of the shoulder. A preliminary report," The Journal of Bone \& Joint Surgery, vol. 62, no. 6, pp. 897-908, 1980.

[5] G. Walch and P. Boileau, "Latarjet-Bristow procedure for recurrent anterior instability," Techniques in Shoulder and Elbow Surgery, vol. 1, no. 4, pp. 256-261, 2000.

[6] T. J. Neviaser, "The anterior labroligamentous periosteal sleeve avulsion lesion: a cause of anterior instability of the shoulder," Arthroscopy: The Journal of Arthroscopic \& Related Surgery, vol. 9, no. 1, pp. 17-21, 1993.

[7] F. P. Tjoumakaris, J. A. Abboud, S. A. Hasan, M. L. Ramsey, G. R. Williams, and G. R. Williams, "Arthroscopic and open Bankart repairs provide similar outcomes," Clinical Orthopaedics and Related Research, vol. 446, pp. 227-232, 2006.

[8] C. Fabbriciani, G. Milano, A. Demontis, S. Fadda, F. Ziranu, and P. D. Mulas, "Arthroscopic versus open treatment of Bankart lesion of the shoulder: a prospective randomized study," Arthroscopy: The Journal of Arthroscopic \& Related Surgery, vol. 20, no. 5, pp. 456-462, 2004.

[9] B. J. Cole, J. L'Insalata, J. Irrgang, and J. J. P. Warner, "Comparison of arthroscopic and open anterior shoulder stabilization," The Journal of Bone and Joint Surgery-American Volume, vol. 82, no. 8, pp. 1108-1114, 2000.

[10] M. R. Green and K. P. Christensen, "Arthroscopic versus open Bankart procedures: a comparison of early morbidity and complications," Arthroscopy: The Journal of Arthroscopic \& Related Surgery, vol. 9, no. 4, pp. 371-374, 1993.

[11] P. Boileau, M. Villalba, J.-Y. Héry, F. Balg, P. Ahrens, and L. Neyton, "Risk factors for recurrence of shoulder instability after arthroscopic Bankart repair," The Journal of Bone \& Joint Surgery, vol. 88, no. 8, pp. 1755-1763, 2006.

[12] S. S. Burkhart and S. M. Danaceau, "Articular arc length mismatch as a cause of failed bankart repair," Arthroscopy: The Journal of Arthroscopic \& Related Surgery, vol. 16, no. 7, pp. 740-744, 2000.

[13] S. S. Burkhart and J. F. De Beer, "Traumatic glenohumeral bone defects and their relationship to failure of arthroscopic Bankart repairs," Arthroscopy: The Journal of Arthroscopic \& Related Surgery, vol. 16, no. 7, pp. 677-694, 2000.

[14] M. Tauber, H. Resch, R. Forstner, M. Raffl, and J. Schauer, "Reasons for failure after surgical repair of anterior shoulder instability," Journal of Shoulder and Elbow Surgery, vol. 13, no. 3, pp. 279-285, 2004.

[15] E. Itoi, S.-B. Lee, L. J. Berglund, L. L. Berge, and K.-N. An, "The effect of a glenoid defect on anteroinferior stability of the shoulder after bankart repair: a cadaveric study," The Journal of Bone and Joint Surgery-American Volume, vol. 82, no. 1, pp. 35-46, 2000.

[16] M. Latarjet, "A propos du traitement des luxations rècidivantes delèpaule," Lyon Chit, vol. 49, pp. 994-1003, 1954.

[17] A. J. Helfet, "Coracoid transplantation for recurring dislocation of the shoulder," The Journal of Bone and Joint Surgery. British Volume, vol. 40-B, no. 2, pp. 198-202, 1958.

[18] J. J. P. Warner, T. J. Gill, J. D. O’Hollerhan, N. Pathare, and P. J. Millett, “Anatomical glenoid reconstruction for recurrent anterior glenohumeral instability with glenoid deficiency using an autogenous tricortical iliac crest bone graft," The American Journal of Sports Medicine, vol. 34, no. 2, pp. 205-212, 2006.

[19] M. T. Provencher, N. Ghodadra, L. LeClere, D. J. Solomon, and A. A. Romeo, "Anatomic osteochondral glenoid reconstruction for recurrent glenohumeral instability with 
glenoid deficiency using a distal tibia allograft," Arthroscopy: The Journal of Arthroscopic \& Related Surgery, vol. 25, no. 4, pp. 446-452, 2009.

[20] A. A. Young, R. Maia, J. Berhouet, and G. Walch, "Open Latarjet procedure for management of bone loss in anterior instability of the glenohumeral joint," Journal of Shoulder and Elbow Surgery, vol. 20, no. 2, pp. S61-S69, 2011.

[21] J. De Beer, S. S. Burkhart, C. P. Roberts, K. Van Rooyen, T. Cresswell, and D. F. Du Toit, "The congruent-arc latarjet," Techniques in Shoulder and Elbow Surgery, vol. 10, no. 2, pp. 62-67, 2009.

[22] C. M. Dolan, S. Hariri, N. D. Hart, and T. R. McAdams, "An anatomic study of the coracoid process as it relates to bone transfer procedures," Journal of Shoulder and Elbow Surgery, vol. 20, no. 3, pp. 497-501, 2011.

[23] N. Ghodadra, A. Gupta, A. A. Romeo et al., "Normalization of glenohumeral articular contact pressures after Latarjet or iliac crest bone-grafting," The Journal of Bone and Joint SurgeryAmerican Volume, vol. 92, no. 6, pp. 1478-1489, 2010.

[24] S. S. Burkhart, J. F. Debeer, A. M. Tehrany, and P. M. Parten, "Quantifying glenoid bone loss arthroscopically in shoulder instability," Arthroscopy: The Journal of Arthroscopic \& Related Surgery, vol. 18, no. 5, pp. 488-491, 2002.

[25] I. K. Y. Lo, P. M. Parten, and S. S. Burkhart, "The inverted pear glenoid: an indicator of significant glenoid bone loss," Arthroscopy: The Journal of Arthroscopic \& Related Surgery, vol. 20, no. 2, pp. 169-174, 2004.

[26] W. H. Montgomery Jr., M. Wahl, C. Hettrich, E. Itoi, S. B. Lippitt, and F. A. Matsen III, "Anteroinferior bonegrafting can restore stability in osseous glenoid defects," The Journal of Bone and Joint Surgery-American Volume, vol. 87, no. 9, pp. 1972-1977, 2005.

[27] D. Patte, J. Bernageau, and P. Bancel, "The anteroinferior vulnerable point of the glenoid rim," in Surgery of the Shoulder, J. E. Bateman and R. P. Welsch, Eds., pp. 94-99, Marcel Dekker, New York, NY, USA, 1985.

[28] D. Patte and J. Debeyre, "Luxations rècidivantes de lèpaule," Encycl Med Chir. Paris-Technique Chirurgicale. Orthop6die, vol. 44265, pp. 4-2, 1980.

[29] J. Allain, D. Goutallier, and C. Glorion, "Long-term results of the latarjet procedure for the treatment of anterior instability of the shoulder," The Journal of Bone \& Joint Surgery, vol. 80, no. 6, pp. 841-852, 1998.

[30] T. B. Edwards, A. Boulahia, and G. Walch, "Radiographic analysis of bone defects in chronic anterior shoulder instability," Arthroscopy: The Journal of Arthroscopic \& Related Surgery, vol. 19, no. 7, pp. 732-739, 2003.

[31] A. S. B. Bankart, "Recurrent or habitual dislocation of the shoulder," BMJ, vol. 2, pp. 1131-1133, 1923.

[32] A. S. B. Bankart, "The pathology and treatment of recurrent dislocation of the shoulder-joint," British Journal of Surgery, vol. 26, no. 101, pp. 23-29, 1938.

[33] G. Perthes, "Über Operationen bei habitueller Schulterluxation," Deutsche Zeitschrift für Chirurgie, vol. 85, no. 1, pp. 199-227, 1906.

[34] C. R. Rowe, D. Patel, and W. W. Southmayd, "The Bankart procedure," The Journal of Bone \& Joint Surgery, vol. 60, no. 1, pp. 1-16, 1978.

[35] T. J. Gill, L. J. Micheli, F. Gebhard, and C. Binder, "Bankart repair for anterior instability of the shoulder. Long-term outcome," The Journal of Bone \& Joint Surgery, vol. 79, no. 6, pp. 850-857, 1997.

[36] K. B. Freedman, A. P. Smith, A. A. Romeo, B. J. Cole, and B. R. Bach, "Open Bankart repair versus arthroscopic repair with transglenoid sutures or bioabsorbable tacks for recurrent anterior instability of the shoulder," The American Journal of Sports Medicine, vol. 32, no. 6, pp. 1520-1527, 2004.

[37] G. M. Gartsman, T. S. Roddey, and S. M. Hammerman, "Arthroscopic treatment of bidirectional glenohumeral instability: two- to five-year follow-up," Journal of Shoulder and Elbow Surgery, vol. 10, no. 1, pp. 28-36, 2001.

[38] S.-H. Kim, K.-I. Ha, Y.-B. Cho, B.-D. Ryu, and I. Oh, "Arthroscopic anterior stabilization of the shoulder," The Journal of Bone and Joint Surgery-American Volume, vol. 85, no. 8, pp. 1511-1518, 2003.

[39] S.-H. Kim, K.-I. Ha, and S.-H. Kim, "Bankart repair in traumatic anterior shoulder instability," Arthroscopy: The Journal of Arthroscopic \& Related Surgery, vol. 18, no. 7, pp. 755-763, 2002.

[40] L. U. Bigliani, P. M. Newton, S. P. Steinmann, P. M. Connor, and S. J. McIlveen, "Glenoid rim lesions associated with recurrent anterior dislocation of the shoulder," The American Journal of Sports Medicine, vol. 26, no. 1, pp. 41-45, 1998.

[41] M. D. Lazarus, J. A. Sidles, D. T. Harryman II, and F. A. Matsen III, "Effect of a chondral-labral defect on glenoid concavity and glenohumeral stability. A cadaveric model," The Journal of Bone \& Joint Surgery, vol. 78, no. 1, pp. 94-102, 1996.

[42] S. R. Montgomery, J. C. Katthagen, J. D. Mikula et al., "Anatomic and biomechanical comparison of the classic and congruent-arc techniques of the latarjet procedure," The American Journal of Sports Medicine, vol. 45, no. 6, pp. 1252-1260, 2017.

[43] J. W. Giles, G. Puskas, M. Welsh, J. A. Johnson, and G. S. Athwal, "Do the traditional and modified Latarjet techniques produce equivalent reconstruction stability and strength?," The American Journal of Sports Medicine, vol. 40, no. 12, pp. 2801-2807, 2012.

[44] W. R. Mook, M. Petri, J. A. Greenspoon, M. P. Horan, G. J. Dornan, and P. J. Millett, "Clinical and anatomic predictors of outcomes after the Latarjet procedure for the treatment of anterior glenohumeral instability with combined glenoid and humeral bone defects," The American Journal of Sports Medicine, vol. 44, no. 6, pp. 1407-1416, 2016.

[45] G. Giacomo, N. de Gasperis, A. De Vita et al., "Coracoid bone graft osteolysis after Latarjet procedure: a comparison study between two screws standard technique vs mini-plate fixation," International Journal of Shoulder Surgery, vol. 7, no. 1, pp. 1-6, 2013. 